\title{
Clinicoradiopathological features and prognosis according to genomic alterations in patients with resected lung adenocarcinoma
}

\author{
Yeonseok Choi ${ }^{1 \#}$, Ki-Hwan Kim ${ }^{2 \#}$, Byeong-Ho Jeong ${ }^{1}$, Kyung-Jong Lee ${ }^{1}$, Hojoong Kim ${ }^{1}$, O. Jung Kwon ${ }^{1}$, \\ Jhingook Kim ${ }^{3}$, Yoon-La Choi ${ }^{4}$, Ho Yun Lee ${ }^{5,6}$, Sang-Won Um ${ }^{1}$
}

${ }^{1}$ Division of Pulmonary and Critical Care Medicine, Department of Medicine, Samsung Medical Center, Sungkyunkwan University School of Medicine, Seoul, South Korea; ${ }^{2}$ Department of Radiology, Myongji Hospital, Goyang, South Korea; ${ }^{3}$ Department of Thoracic and Cardiovascular Surgery, Samsung Medical Center, Sungkyunkwan University School of Medicine, Seoul, South Korea; ${ }^{4}$ Department of Pathology and Translational Genomics, Samsung Medical Center, Sungkyunkwan University School of Medicine, Seoul, South Korea; Department of Radiology and Center for Imaging Science, Samsung Medical Center, Sungkyunkwan University School of Medicine, Seoul, South Korea; ${ }^{6}$ Department of Health Sciences and Technology, SAIHST, Sungkyunkwan University, Seoul, South Korea

Contributions: (I) Conception and design: SW Um; (II) Administrative support: Y Choi; (III) Provision of study materials or patients: Y Choi; (IV) Collection and assembly of data: All authors; (V) Data analysis and interpretation: Y Choi, KH Kim, HY Lee, SW Um; (VI) Manuscript writing: All authors; (VII) Final approval of manuscript: All authors.

\#These authors contributed equally to this work.

Correspondence to: Sang-Won Um. Division of Pulmonary and Critical Care Medicine, Department of Medicine, Samsung Medical Center, Sungkyunkwan University School of Medicine, 81 Irwon-ro, Gangnam-gu, Seoul 06351, South Korea. Email: sangwonum@skku.edu; Ho Yun Lee. Department of Radiology and Center for Imaging Science, Samsung Medical Center, Sungkyunkwan University School of Medicine, 81 Irwon-ro, Gangnam-gu, Seoul 06351, South Korea; Department of Health Sciences and Technology, SAIHST, Sungkyunkwan University, Seoul 06351, South Korea. Email: hoyunlee96@gmail.com.

Background: We investigated the clinicoradiopathological features and prognosis according to genomic alterations in patients with surgically resected lung adenocarcinoma.

Methods: Patients who underwent surgical resection for pathologic stage I, II, or IIIA lung adenocarcinoma between 2009 and 2016 and for whom results regarding EGFR mutation, ALK immunohistochemistry (IHC), and KRAS mutation were available were included. Clinicoradiopathological characteristics, genomic alterations, and disease-free survival were analyzed retrospectively.

Results: Of 164 patients, 86 (52.4\%) were female and 94 (57.3\%) were never-smokers. The most common imaging patterns were part-solid lesion (67.7\%) followed by solid (26.2\%) and non-solid (6.1\%) lesions. EGFR mutation, ALK IHC, and KRAS mutation were positive in 95 (57.9\%), 9 (5.5\%), and 11 (6.7\%) patients, respectively. EGFR mutation positivity was associated with female sex, never-smoker, subsolid pattern on radiological examination, and acinar or papillary predominant histologic subtype. ALK IHC positivity was associated with longer maximal diameter, advanced stage, solid pattern on radiological examination, solid predominant histologic subtype, and distant metastasis during follow-up. KRAS mutation positivity was associated with male sex, smoker, solid pattern on radiological examination, and invasive mucinous adenocarcinoma on histologic analysis. In multivariable analysis, ALK IHC positivity and lymph node involvement were independently associated with recurrence. However, solidity was not an independent risk factor for recurrence.

Conclusions: Genomic alterations are associated with clinicoradiopathologic features in patients with resected lung adenocarcinoma. Identifying genomic alterations could help to predict the prognosis of earlystage lung adenocarcinoma.

$\wedge$ ORCID: 0000-0002-9005-4525. 
Keywords: Lung adenocarcinoma; solidity; epidermal growth factor receptor (EGFR); anaplastic lymphoma kinase (ALK); Kirsten rat sarcoma viral oncogene homolog (KRAS)

Submitted Apr 22, 2020. Accepted for publication Sep 14, 2020.

doi: $10.21037 /$ jtd-20-1716

View this article at: http://dx.doi.org/10.21037/jtd-20-1716

\section{Introduction}

With advances in genomic analysis, various genomic alterations that contribute to the development of cancer have been identified. Targeting these genomic alterations has become an important therapeutic option in patients with neoplasms, and this approach is changing the therapeutic paradigm (1). In lung adenocarcinoma, epidermal growth factor receptor (EGFR) gene mutation, anaplastic lymphoma kinase $(A L K)$ gene rearrangement, and Kirsten rat sarcoma viral oncogene homolog (KRAS) gene mutation are the most frequently detected genomic alterations (2). Recent studies demonstrated that agents targeting EGFR mutation or $A L K$ rearrangement, including EGFR tyrosine-kinase inhibitors (TKIs) and ALK TKIs, respectively, increased the response rate and progressionfree survival in patients with lung adenocarcinoma (3-5). However, there are no approved target agents for cases with $K R A S$ mutation. Instead, the presence of a KRAS mutation can indicate a poor prognosis with regard to survival and be a predictor of a poor response to EGFR TKIs (6).

In terms of the radiological evaluation of lung adenocarcinoma, computed tomography (CT) is a costeffective and non-invasive tool in the diagnosis, treatment, and follow-up of lung cancer. Since the introduction of lowdose spiral CT as a screening tool, the early diagnosis of lung cancer has increased $(7,8)$. Particularly, early-stage lung adenocarcinoma may be detected on CT as solid or subsolid nodules, which can be further classified as non-solid or part-solid nodules (9). A higher proportion of ground-glass opacity (GGO) is correlated with a better prognosis, and recent guidelines recommended the management of nodules according to their solidity (10-12). Recently, it was reported that EGFR mutation was associated with GGO and $A L K$ rearrangement was shown to present a solid pattern on chest CT $(13,14)$. However, the associations of radiological features and prognosis in relation to genomic alterations in early-stage lung adenocarcinoma remain uncertain. Therefore, we investigated the clinicoradiopathological features and prognosis according to genomic alterations in patients with stage I-IIIA resected lung adenocarcinoma.
We present the following article in accordance with the STROBE reporting checklist (available at http://dx. doi. org/10. 21037/jtd-20-1716).

\section{Methods}

\section{Patient selection and clinical assessment}

Patients who underwent surgical resection for pathologic stage I, II, or IIIA lung adenocarcinoma at Samsung Medical Center between 2009 and 2016 and for whom results regarding EGFR mutation, ALK immunohistochemistry (IHC), and KRAS mutation were available were included in this retrospective observational study. Patients who had undergone treatment for lung cancer before surgery, such as chemotherapy or radiotherapy, which could alter the pathological results were excluded. Patient characteristics, including age, sex, smoking status, surgical procedure type, recurrence, and outcomes were obtained from medical records.

The study was conducted in accordance with the Declaration of Helsinki (as revised in 2013). This study was approved by the Institutional Review Board of Samsung Medical Center (IRB No. SMC 2018-03-125) and individual consent for this retrospective analysis was waived. All data were anonymized and de-identified prior to analysis.

\section{Radiological evaluation}

All selected patients underwent chest CT within 2 months prior to surgery. While CT examinations were performed at multiple institutions with variable protocols, thin-section chest CT images that could indicate non-solid lesions based on the consensus of two experienced radiologists were reviewed. They independently measured diameters and categorized tumors according to CT features: solidity (non-solid, part-solid, and solid), presence of spiculation, lobulation, pleural tagging, air-bronchogram, and bubble lucency. Non-solid lesions were characterized by hazy increased attenuation that did not obliterate the bronchial 
and vascular margins, part-solid lesions were those with both GGO and soft tissue attenuation, and solid lesions were defined as those with homogeneous soft tissue attenuation $(9,15)$.

The extent of GGO in each lesion was evaluated quantitatively using the GGO proportion. For each entire tumor and its solid portion, regions of interest were delineated on the axial images to generate a volume of interest with a semiautomated approach. The longest diameter was estimated from three-dimensional tumor measurements of regions of interest. The maximal diameter of the lesion was multiplied by its longest perpendicular diameter to obtain the total area on lung windows. To calculate the solid area on lung windows, the maximal diameter of the solid portion was multiplied by its longest perpendicular diameter. GGO proportion was defined as follows:

GGO proportion $(\%)=100 \times[1-($ solid area on lung windows)/(total area on lung windows)].

\section{Histopathological evaluation}

Pathologic tumor, node and metastasis (TNM) staging was performed following the International Association for the Study of Lung Cancer (IASLC) proposals for the $8^{\text {th }}$ edition of TNM classification for lung cancer (16). For histological subtyping, we classified lesions following the 2011 International Association for the Study of Lung Cancer/American Thoracic Society/European Respiratory Society (IASLC/ATS/ERS) histologic classification of lung adenocarcinoma categorized according to the predominant histologic subtype (17).

\section{EGFR and KRAS mutations and ALK IHC}

To evaluate EGFR and KRAS gene mutations, extracted genomic DNA was isolated from formalin-fixed paraffinembedded (FFPE) tissue. DNA sequencing for $E G F R$ mutations in exons 18, 19,20, and 21 was performed using real-time polymerase chain reaction (PCR) and a peptide nucleic acid (PNA) clamping EGFR Mutation Detection Kit (Panagene, Inc., Daejeon, Korea). KRAS mutations in exons 12 and 13 were evaluated by Sanger sequencing. ALK protein expression was evaluated by IHC (1:40, NCL-ALK, clone 5A4, Novocastra, Newcastle upon Tyne, UK) with FFPE tissue. Diffuse and strong cytoplasmic positivity of tumor cells was considered positive for ALK IHC (18). Although it was well known that there was a good correlation between the results of ALK IHC and fluorescence in situ hybridization (FISH) (19), ALK discordant (IHC-positive/FISH-negative) cases were also reported $(20,21)$. In the previous studies, $A L K$ discordant (IHC-positive/FISH-negative) cases showed $A L K$ gene amplification (20) and responsiveness to ALK-TKI (21). In this study, ALK IHC positivity was regarded as a surrogate marker for $A L K$ gene rearrangement or amplification (19).

\section{Statistical analysis}

For descriptive statistics, the means with standard deviation or medians with interquartile range (IQR) were used for continuous variables. Frequencies with percentages were presented for categorical variables. Continuous variables were analyzed by one-way analysis of variance, Kruskal-Wallis test, or Mann-Whitney $\mathrm{U}$ test. To evaluate categorical variables, Pearson's $\chi^{2}$ test or Fisher's exact test was used. For post hoc analysis, the Mann-Whitney $\mathrm{U}$ test, Pearson's $\chi^{2}$ test, Fisher's exact test, and Bonferroni's correction were performed.

The Kaplan-Meier method was used to estimate diseasefree survival, which was assessed from the date of surgery to the date of diagnosis of first recurrence on medical records. The log-rank test was performed to identify differences in disease-free survival according to genomic alterations and solidity. Multivariable analyses with Cox proportionalhazards regression and stepwise selection were carried out using variables with $\mathrm{P}<0.05$ on univariable analysis and clinically relevant variables (age, sex, and smoking status) to identify risk factors for recurrence after surgery. During stepwise variable selection, variables with $\mathrm{P}<0.10$ on univariable analysis and clinically relevant variables were entered and those with $\mathrm{P} \geq 0.05$ were removed (22). Firth's penalized maximum likelihood estimation method was implemented due to rare events in Cox regression model. With $95 \%$ confidence intervals (CIs), $\mathrm{P}<0.05$ was taken to indicate statistical significance. Statistical analyses were performed using SAS version 9.4 (SAS Institute, Cary, NC, USA), R 3.5.1 (R Foundation for Statistical Computing, Vienna, Austria; http://www.R-project.org), and IBM SPSS 25.0 (IBM Corp., Armonk, NY, USA).

\section{Results}

\section{Baseline characteristics}

A total of 164 patients with surgically resected lung 


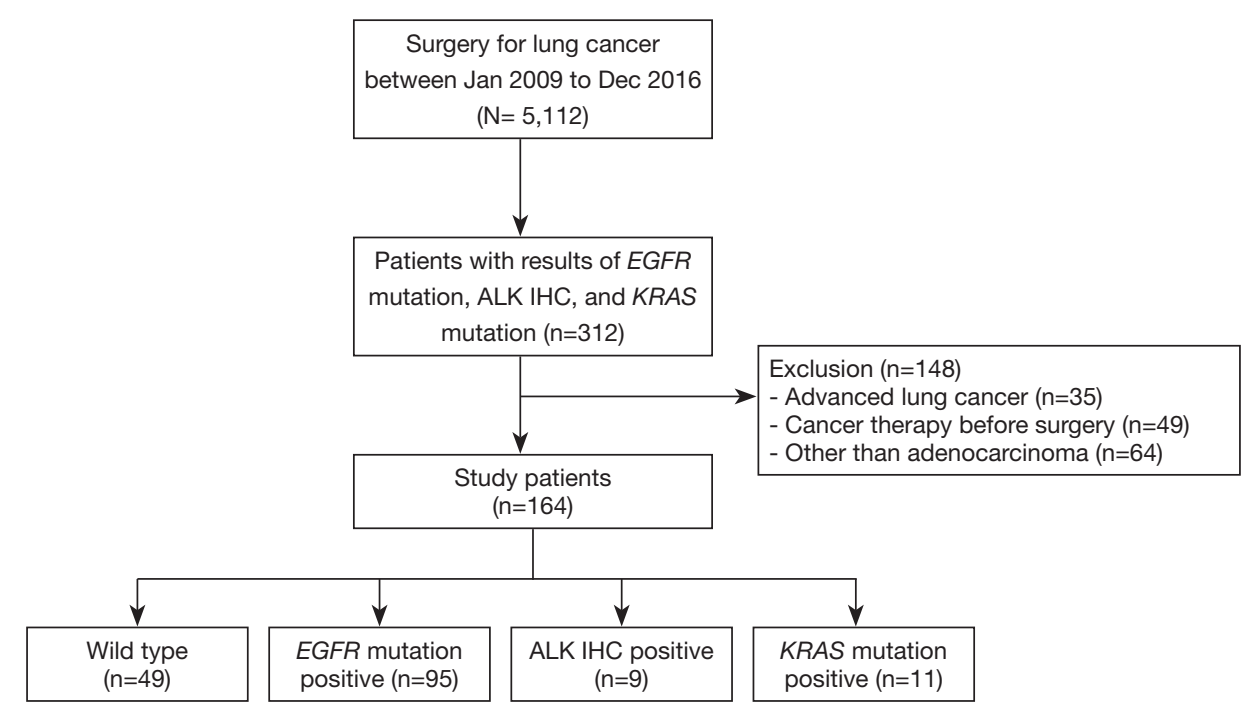

Figure 1 Flow diagram of subject enrollment.

adenocarcinoma were included in analysis (Figure 1) and the baseline characteristics of these patients are summarized in Table 1. The mean age was 60.7 years, 86 patients $(52.4 \%)$ were female, and 94 patients $(57.3 \%)$ were never-smokers. The most common imaging patterns were part-solid lesion (67.7\%) followed by solid lesion (26.2\%) and non-solid lesion $(6.1 \%)$. With regard to surgical procedure, 122 patients $(74.4 \%)$ underwent lobectomy, and limited resection including segmentectomy or wedge resection was performed in 42 patients $(25.6 \%)$. All patients included in this study achieved R0 resection and 158 patients (96.3\%) received lymph node dissection or sampling. Most patients had a diagnosis of pathologic stage I (79.9\%) lung adenocarcinoma followed by stage II (11.0\%) and stage IIIA (9.1\%). Acinar predominant subtype $(68.3 \%)$ was the most common histologic subtype followed by solid predominant subtype (7.9\%) and papillary predominant subtype $(6.7 \%)$. The median follow-up period after surgery was 50.0 (IQR, 29.553.0) months. Recurrences developed in 32 patients (19.5\%) during the follow-up period. Among these patients, 2 patients (1.2\%) had only locoregional relapse; 26 patients (15.9\%) had distant metastasis alone; and the remainder (4 patients, $2.4 \%$ ) had both locoregional relapse and distant metastasis (Table 1).

\section{Genomic alterations}

With regard to genomic alterations, patients had EGFR mutation (95/164, 57.9\%), ALK IHC positivity (9/164, $5.5 \%)$, and KRAS mutation (11/164, 6.7\%). In terms of subtypes of EGFR mutation, 47 patients (47/95, 49.5\%) were positive for the L858R point mutation and 41 patients (41/95, $43.2 \%)$ were positive for an exon 19 deletion. Patients with ALK IHC positivity had an IHC score of $2(6 / 9,66.6 \%)$ or $3(3 / 9,33.3 \%)$, and all patients with a $K R A S$ mutation had a missense mutation in codon 12 (11/11, 100\%) (Table 2).

\section{Analysis of clinicoradiological and pathological features in relation to genomic alterations}

Analyses of the characteristics of each nodule according to genomic alterations indicated that $E G F R$ mutation was significantly associated with female sex $(\mathrm{P}=0.001)$, neversmokers $(\mathrm{P}=0.001)$, part-solid lesions on radiological examination $(\mathrm{P}<0.001)$, and acinar or papillary predominant subtype on histological analysis $(\mathrm{P}<0.001)$. ALK IHC positivity was associated with longer maximal diameter $(\mathrm{P}=0.012)$, solid lesions on radiological examination $(\mathrm{P}<0.001)$, advanced pathologic stage $(\mathrm{P}=0.009)$, solid predominant subtype on histological analysis $(\mathrm{P}<0.001)$, and distant metastasis during follow-up $(\mathrm{P}<0.001)$. KRAS mutation positivity was associated with male sex $(\mathrm{P}=0.001)$, smokers $(\mathrm{P}=0.001)$, solid lesions on radiological examination $(\mathrm{P}<0.001)$, and invasive mucinous adenocarcinoma on histological analysis $(\mathrm{P}<0.001)$ (Table 3).

\section{Radiological morphology and genomic alterations}

In terms of subsolid and solid lesions according to genomic 
Table 1 Baseline characteristics

\begin{tabular}{|c|c|}
\hline Variables & Number \\
\hline Age (years) & $60.7 \pm 8.9$ \\
\hline Sex, female, n (\%) & $86(52.4)$ \\
\hline Never-smoker, n (\%) & $94(57.3)$ \\
\hline \multicolumn{2}{|l|}{ Imaging pattern, n (\%) } \\
\hline Non-solid & $10(6.1)$ \\
\hline Part-solid & $111(67.7)$ \\
\hline Solid & $43(26.2)$ \\
\hline \multicolumn{2}{|l|}{ Surgery, n (\%) } \\
\hline \multicolumn{2}{|l|}{ Extent of resection } \\
\hline Wedge resection & $18(11.0)$ \\
\hline Segmentectomy & $24(14.6)$ \\
\hline Lobectomy & $122(74.4)$ \\
\hline $\begin{array}{l}\text { Mediastinal lymph node dissection or } \\
\text { sampling }^{\dagger}\end{array}$ & $158(96.3)$ \\
\hline $\mathrm{R} 0$ resection & $164(100.0)$ \\
\hline \multicolumn{2}{|l|}{ Pathologic stage $\mathrm{e}^{\ddagger}, \mathrm{n}(\%)$} \\
\hline Stage I & $131(79.9)$ \\
\hline Stage II & $18(11.0)$ \\
\hline Stage III & $15(9.1)$ \\
\hline \multicolumn{2}{|l|}{ Histologic subtype ${ }^{\S}, \mathrm{n}(\%)$} \\
\hline Nonmucinous MIA & $6(3.7)$ \\
\hline \multicolumn{2}{|l|}{ Invasive adenocarcinoma } \\
\hline Lepidic predominant & $8(4.9)$ \\
\hline Acinar predominant & $112(68.3)$ \\
\hline Papillary predominant & $11(6.7)$ \\
\hline Micropapillary predominant & $1(0.6)$ \\
\hline Solid predominant & $13(7.9)$ \\
\hline Invasive mucinous adenocarcinoma & $7(4.3)$ \\
\hline Enteric & $2(1.2)$ \\
\hline Unclassified & $4(2.4)$ \\
\hline Follow-up duration after surgery (months) & $50.0(29.5-53.0)$ \\
\hline Recurrence, n (\%) & $32(19.5)$ \\
\hline Locoregional relapse & $6(3.7)$ \\
\hline Ipsilateral lung & $1(0.6)$ \\
\hline Regional lymph node & $5(3.0)$ \\
\hline
\end{tabular}

Table 1 (Continued)
Table 1 (Continued)

\begin{tabular}{lc}
\hline Variables & Number \\
\hline Distant metastasis & $30(18.3)$ \\
Contralateral lung & $13(7.9)$ \\
Pleura & $7(4.3)$ \\
Brain & $7(4.3)$ \\
Bone & $6(3.7)$ \\
Liver & $1(0.6)$ \\
Kidney & $1(0.6)$ \\
\hline
\end{tabular}

Data are presented as the mean \pm standard deviation, median (IQR), or number (\%). ${ }^{\dagger}$, mediastinal lymph node dissection was performed in 145 patients $(88.4 \%) ;{ }^{\ddagger}$, IASLC $8^{\text {th }}$ edition of the TNM classification for lung cancer; ${ }^{\S}, 2011$ IASLC/ATS/ERS histologic classification of lung adenocarcinoma. MIA, minimally invasive adenocarcinoma.

alterations, ALK IHC positivity was associated with solid lesions compared to patients positive for EGFR mutation $(\mathrm{P}<0.001)$ or wild type $(\mathrm{P}=0.006)$ (Figure $2 A)$. ALK IHC positivity was related to a lower GGO proportion on chest CT compared to positive EGFR mutation $(\mathrm{P}=0.012)$ or wild type $(\mathrm{P}=0.030)$ (Figure $2 B)$.

\section{Risk factors for recurrence after surgery}

In univariable analysis, solid lesion, ALK IHC positivity, non-lepidic or acinar predominant subtype, pathologic T2-4 stage, lymph node involvement, and administration of adjuvant chemotherapy were risk factors for recurrence. However, the administration of adjuvant chemotherapy could be related to advanced stage of the disease and was excluded from the multivariable analysis to avoid collinearity. In multivariable analysis, ALK IHC positivity $(\mathrm{HR}=2.750,95 \% \mathrm{CI}, 1.097-6.897 ; \mathrm{P}=0.031)$ and lymph node involvement $(\mathrm{HR}=7.594,95 \% \mathrm{CI}, 3.497-16.487$; $\mathrm{P}<0.001)$ were independent risk factors for recurrence (Table 4). The disease-free survival rate according to genomic alterations was significantly lower in patients with ALK IHC positivity than in those with other genomic alterations $(\mathrm{P}<0.001)$; the median disease-free survival was 24.0 months (95\% CI, 15.2-32.8) in patients with ALK IHC positivity and not reached in those with other genomic alterations (Figure 3). 
Table 2 Summary of genomic alterations in patients with lung adenocarcinoma

\begin{tabular}{lc}
\hline Variables & Number, $\mathrm{n}(\%)$ \\
\hline EGFR mutation & \\
Wild type & $69 / 164(42.1)$ \\
Mutation positive & $95 / 164(57.9)$ \\
$\quad$ Exon 18 G719X point mutation & $2 / 95(2.1)$ \\
Exon 19 deletion & $41 / 95(43.2)$ \\
Exon 20 insertion & $1 / 95(1.1)$ \\
Exon 21 L858R point mutation & $47 / 95(49.5)$ \\
Exon 21 L858R or L861Q point mutation & $3 / 95(3.2)$ \\
Exon 21 L858R and K860I point mutation & $1 / 95(1.1)$ \\
ALK IHC & \\
Negative & $155 / 164(94.5)$ \\
Positive & $9 / 164(5.5)$ \\
IHC 2+ & $6 / 9(66.7)$ \\
IHC 3+ & $3 / 9(33.3)$ \\
KRAS mutation & $11 / 164(6.7)$ \\
Wild type & $11 / 11(100.0)$ \\
Mutation positive & \\
Missense in codon 12 & \\
\hline
\end{tabular}

$E G F R$, epidermal growth factor receptor gene; ALK, anaplastic lymphoma kinase; IHC, immunohistochemistry; KRAS, Kirsten rat sarcoma viral oncogene homolog gene.

\section{Discussion}

Our data suggest that EGFR mutation is associated with part-solid or subsolid lesions on radiological examination and acinar or papillary predominant subtype on histological analysis. ALK IHC positivity was detected more frequently in patients with large or advanced stage tumors, solid lesions on radiological examination, solid predominant subtype on histological analysis, and distant metastasis during follow-up. KRAS mutation was related to solid lesions on radiological examination and invasive mucinous adenocarcinoma on histological analysis. With regard to disease-free survival, ALK IHC positivity and lymph node involvement were risk factors for recurrence.

Previous studies yielded inconsistent results regarding the association between EGFR mutation and the presence of GGO patterns. Most studies reported that EGFR mutation was linked to lesions with a higher proportion of GGO $(23,24)$. However, Glynn et al. (25) reported no significant correlation between $E G F R$ mutation and a GGO component. Two other studies indicated that EGFR mutation was more common in lesions with a $>50 \%$ solid component in comparison to cases with a lower solid proportion (26,27). In the present study, EGFR mutation was associated with a higher GGO percentage compared to ALK IHC positivity or KRAS mutation. Additionally, EGFR mutation was detected more frequently in part-solid or subsolid lesions, which were partly composed of GGO components. These discrepancies between studies may have been mediated by the prevalence of EGFR mutation in the study population or use of different methods for quantification and classification of the GGO component. In terms of histological features of EGFR mutation, no significant findings have been reported with papillary predominant subtype, but Wang et al. (28) reported that patients with lepidic or acinar predominant adenocarcinoma tended to have an EGFR mutation. These findings were partially inconsistent with our results, which may have been due to the inclusion of advanced lung adenocarcinoma patients with various manifestations (29).

Findings from previous studies regarding the correlation between ALK-positive lung cancer and its GGO component suggested that ALK-positive lesions were more likely to present with solid components on CT, consistent with our results $(13,30)$. However, a study of lung cancer from early to advanced stages reported that lesions $<20 \mathrm{~mm}$ in size were more likely to be $A L K$ fusion-positive tumors (31). With regard to histological features, Wang et al. (28) reported that $A L K$ rearrangement was associated with solid predominant subtype, as shown in our study. For ALK IHC, in this study, 7 of 9 IHC-positive cases (2+ or 3+) were further evaluated by $A L K$ FISH at the time of recurrence. Of 7 cases examined, 6 cases were positive for $A L K$ FISH ( $>15 \%$ rearranged signals). However, one case positive for ALK IHC (3+) was negative for $A L K$ FISH and received cytotoxic chemotherapy instead of ALK-TKI due to a financial issue. Unfortunately, the patient expired due to the progression of the disease. In the previous studies, $A L K$ discordant (IHC-positive/FISH-negative) cases were related to $A L K$ gene amplification (20) and 13 (100\%) of 13 discordant (IHC-positive/FISH-negative) cases responded to ALK-TKI (21). Therefore, the discrepancies between IHC and FISH data seem to be associated with biological events rather technical issues. Recent NCCN guidelines also acknowledged FDA-approved ALK IHC (D5F3 CDx Assay) as a stand-alone test, not requiring confirmation by 
Table 3 Clinicoradiopathological features according to genomic alterations

\begin{tabular}{|c|c|c|c|c|c|}
\hline Variables & $\begin{array}{l}\text { Wild type }(n=49), \\
n(\%)\end{array}$ & $\begin{array}{c}\text { EGFR Mutation } \\
\text { positive }(n=95), n(\%)\end{array}$ & $\begin{array}{l}\text { ALK IHC positive } \\
(n=9), n(\%)\end{array}$ & $\begin{array}{c}\text { KRAS Mutation } \\
\text { positive }(n=11), n(\%)\end{array}$ & $P$ value \\
\hline Age (years) & $61.1 \pm 8.5$ & $59.8 \pm 8.0$ & $64.7 \pm 14.1$ & $63.3 \pm 13.0$ & 0.298 \\
\hline Sex, female & $18(36.7)$ & $62(65.3)$ & $4(44.4)$ & $2(18.2)$ & 0.001 \\
\hline Never-smoker & $22(44.9)$ & $66(69.5)$ & $4(44.4)$ & $2(18.2)$ & 0.001 \\
\hline Non-solid & $4(8.2)$ & $4(4.2)$ & $0(0.0)$ & $2(18.2)$ & \\
\hline Part-solid & $32(65.3)$ & $74(77.9)$ & $1(11.1)$ & $4(36.4)$ & \\
\hline Solid & $13(26.5)$ & $17(17.9)$ & 8 (88.9) & $5(45.5)$ & \\
\hline Air-bronchogram* & $21(42.9)$ & $57(60.0)$ & $1(11.1)$ & $6(54.5)$ & 0.016 \\
\hline Bubble lucency & 7 (14.3) & $18(18.9)$ & $0(0.0)$ & $2(18.2)$ & 0.593 \\
\hline Pathologic stage $^{\dagger}$ & & & & & 0.009 \\
\hline Stage I & $43(87.8)$ & $77(81.1)$ & $4(44.4)$ & $7(63.6)$ & \\
\hline Stage II & $2(4.1)$ & $9(9.5)$ & $3(33.3)$ & $4(36.4)$ & \\
\hline Stage III & $4(8.2)$ & $9(9.5)$ & $2(22.2)$ & $0(0.0)$ & \\
\hline Histologic subtype ${ }^{\ddagger}$ & & & & & $<0.001$ \\
\hline Micropapillary predominant & $0(0.0)$ & $0(0.0)$ & $0(0.0)$ & $1(9.1)$ & \\
\hline Solid predominant & $3(6.1)$ & $5(5.3)$ & $4(44.4)$ & $1(9.1)$ & \\
\hline Invasive mucinous & $3(6.1)$ & $0(0.0)$ & $0(0.0)$ & $4(36.4)$ & \\
\hline Enteric predominant & $2(4.1)$ & $0(0.0)$ & $0(0.0)$ & $0(0.0)$ & \\
\hline Unclassified & $1(2.0)$ & $0(0.0)$ & 3 (33.3) & $0(0.0)$ & \\
\hline Recurrence & & & & & $<0.001$ \\
\hline Locoregional relapse & $0(0.0)$ & $0(0.0)$ & $2(22.2)$ & $0(0.0)$ & \\
\hline Distant metastasis & $3(6.1)$ & $16(16.8)$ & $5(55.6)$ & $2(18.2)$ & \\
\hline Locoregional relapse and distant metastasis & $0(0.0)$ & $4(4.2)$ & $0(0.0)$ & $0(0.0)$ & \\
\hline
\end{tabular}

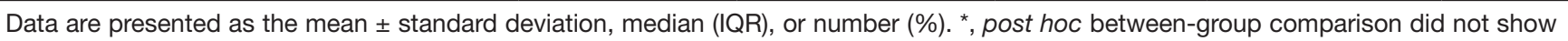
a significant difference; ${ }^{\dagger}$, IASLC $8^{\text {th }}$ edition of the TNM classification for lung cancer; ${ }^{\ddagger}, 2011$ IASLC/ATS/ERS histologic classification of lung adenocarcinoma. EGFR, epidermal growth factor receptor gene; ALK, anaplastic lymphoma kinas; IHC, immunohistochemistry; KRAS, Kirsten rat sarcoma viral oncogene homolog gene; MIA, minimally invasive adenocarcinoma; Invasive mucinous, invasive mucinous adenocarcinoma. 

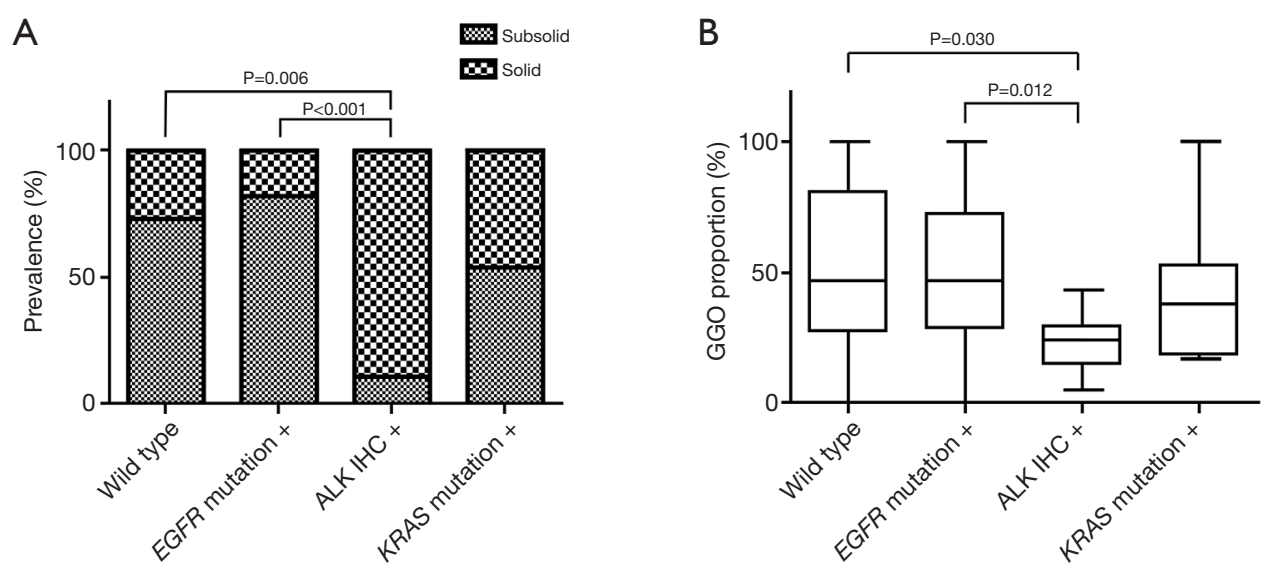

Figure 2 Solidity according to genomic alterations. (A) Prevalence of subsolid and solid lesions according to genomic alteration. (B) Ground-glass opacity (GGO) proportion and genomic alterations. EGFR, epidermal growth factor receptor gene; ALK, anaplastic lymphoma kinase; IHC, immunohistochemistry; KRAS, Kirsten rat sarcoma viral oncogene homolog gene.

Table 4 Risk factors for recurrence in patients with resected lung adenocarcinoma

\begin{tabular}{|c|c|c|c|c|c|c|}
\hline Variables & \multicolumn{3}{|c|}{ Univariable } & \multicolumn{3}{|c|}{ Multivariable } \\
\hline Age ( $\geq 61$ vs. $<61$ years) & 1.375 & $0.686-2.754$ & 0.369 & & & \\
\hline Sex (female vs. male) & 0.752 & $0.375-1.505$ & 0.421 & & & \\
\hline Smoking status (current/former vs. never) & 1.315 & $0.656-2.633$ & 0.440 & & & \\
\hline Surgery (lobectomy vs. limited resection) $)^{\dagger \ddagger}$ & 3.717 & $0.704-19.616$ & 0.122 & & & \\
\hline ALK IHC (positive vs. negative) & 6.926 & $3.004-15.965$ & $<0.001$ & 2.750 & $1.097-6.897$ & 0.031 \\
\hline Histologic subtype (others vs. lepidic or acinar) & 2.772 & $1.384-5.553$ & 0.004 & & & \\
\hline Pathologic T stage (T2-4 vs. T1) & 3.797 & $1.856-7.766$ & $<0.001$ & & & \\
\hline
\end{tabular}

${ }^{\dagger}$, lobectomy vs. limited resection (wedge resection or segmentectomy). ${ }^{\ddagger}$, hazard ratios could not be calculated in the multivariable analysis as only one case of limited resection showed evidence of recurrence. HR, hazard ratio; Cl, confidence interval; ALK, anaplastic lymphoma kinase; IHC, immunohistochemistry.

\section{FISH (32).}

Several studies have reported radiological features of $K R A S$-mutant lung adenocarcinoma (26,33). Although there were only two patients in their study, Chung et al. (26) detected a KRAS mutation in GGO lesions with $>50 \%$ solid component. On the other hand, Rizzo et al. (33) found no significant difference in rate of KRAS mutation positivity between solid and subsolid lesions. In this study, we applied a different classification method for pulmonary lesions (i.e., non-solid, part-solid, or solid lesions) and showed that $K R A S$ mutation was associated with solid lesions compared to EGFR mutation.

The characteristics of wild type in this study were similar to those of cases with EGFR mutation, especially with regard to solidity and histologic subtype. PNA clamping is a more sensitive method for the detection of EGFR mutations compared to direct sequencing (34). However, the similarities between EGFR mutation and wild type may 


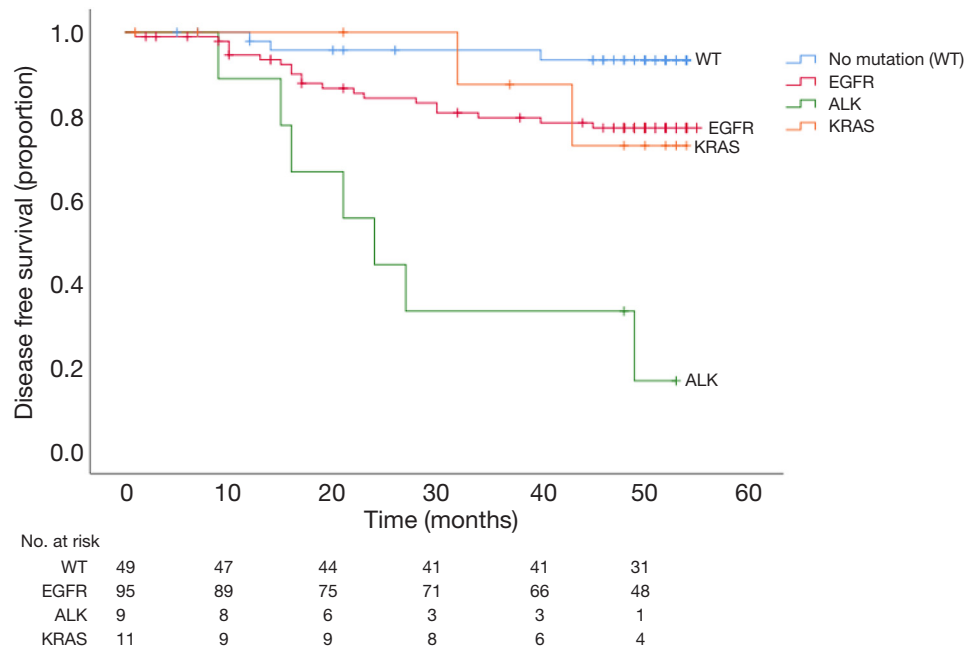

Figure 3 Disease-free survival according to genomic alterations $(\mathrm{P}<0.001$; wild type, EGFR mutation, ALK IHC, and KRAS mutation). WT, wild type; $E G F R$, epidermal growth factor receptor gene; ALK, anaplastic lymphoma kinase; KRAS, Kirsten rat sarcoma viral oncogene homolog gene.

result from false-negative results for EGFR mutation. For example, $64.5 \%$ (78/121) of subsolid lesions were classified as having an EGFR mutation in the present study. On the other hand, in our previous study, about $90 \%$ of lung adenocarcinoma with subsolid morphology were classified as having an EGFR mutation based on next-generation sequencing, which is more sensitive than PNA clamping or direct sequencing (35). In addition, wild type in this study could have included other driver mutations, such as ROS protooncogene 1 (ROS1) rearrangements or B-Raf protooncogene $(B R A F)$ point mutations.

Our observations indicate that lymph node metastasis was a risk factor for recurrence, which is consistent with previous studies (36,37). We also demonstrated that ALK IHC positivity was independently associated with poor disease-free survival. ALK IHC positivity was associated with more distant metastasis during follow-up (Table 3). Even in patients with stage IA or IB disease, recurrence occurred in $50 \%(2 / 4)$ of those positive for ALK IHC. Therefore, the benefits of adjuvant chemotherapy according to genomic alterations should be evaluated for patients with pathologic stage I lung adenocarcinoma in future studies. To the best of our knowledge, this is the first study to show differences in disease-free survival according to genomic alterations in patients with resected lung adenocarcinoma. Not only for metastatic lung cancer, but genomic evaluation in early-stage lung cancer may help in predicting prognosis, such as disease-free survival.
This study has several limitations. First, it was a retrospective single-center study in a population of $100 \%$ Asian patients with a relatively small sample size. As we included only patients for whom EGFR mutation, ALK IHC, and KRAS mutation results were available, the possibility of potential selection bias should be considered. Therefore, the results of our study should be interpreted conservatively. Second, chest CT was performed at various institutions with different protocols or thicknesses. However, experienced radiologists independently reviewed each CT scan for quality control. Third, only early-stage lung adenocarcinoma was included in the study; it is unclear whether the results of this study would be applicable to advanced lung adenocarcinoma.

\section{Conclusions}

Genomic alterations are associated with clinicoradiopathologic features in patients with resected lung adenocarcinoma. Identifying genomic alterations could help to predict the prognosis of early-stage lung adenocarcinoma.

\section{Acknowledgments}

Funding: This work was supported by the National Research Foundation of Korea (NRF) grant funded by the Korea government (MSIT) (2019R1A4A1028268), and by the 
Korea Health Technology R\&D Project through the Korea Health Industry Development Institute (KHIDI), which was funded by the Ministry of Health \& Welfare (HI17C0086) and the National Research Foundation of Korea (NRF) grant funded by the Korean government (MSIP; Ministry of Science, ICT \& Future Planning) (No. NRF2016R1A2B4013046 and NRF-2017M2A2A7A02018568). The funders of the study had no role in study design, data collection, data analysis, data interpretation, or writing of the report.

\section{Footnote}

Reporting Checklist: The authors have completed the STROBE reporting checklist. Available at http://dx. doi. org/10. 21037/jtd-20-1716

Data Sharing Statement: Available at http://dx. doi. org/10. 21037/jtd-20-1716

Peer Review File: Available at http://dx.doi.org/10.21037/jtd20-1716

Conflicts of Interests: All authors have completed the ICMJE uniform disclosure form (available at http://dx. doi. org/10. 21037/jtd-20-1716). The authors have no conflicts of interest to declare.

Ethical Statement: The authors are accountable for all aspects of the work in ensuring that questions related to the accuracy or integrity of any part of the work are appropriately investigated and resolved. The study was conducted in accordance with the Declaration of Helsinki (as revised in 2013). The Institutional Review Board of Samsung Medical Center approved this retrospective study (IRB No. SMC 2018-03-125) and individual consent for this retrospective analysis was waived.

Open Access Statement: This is an Open Access article distributed in accordance with the Creative Commons Attribution-NonCommercial-NoDerivs 4.0 International License (CC BY-NC-ND 4.0), which permits the noncommercial replication and distribution of the article with the strict proviso that no changes or edits are made and the original work is properly cited (including links to both the formal publication through the relevant DOI and the license). See: https://creativecommons.org/licenses/by-ncnd/4.0/.

\section{References}

1. Buettner R, Wolf J, Thomas RK. Lessons learned from lung cancer genomics: the emerging concept of individualized diagnostics and treatment. J Clin Oncol 2013;31:1858-65.

2. Kris MG, Johnson BE, Berry LD, et al. Using multiplexed assays of oncogenic drivers in lung cancers to select targeted drugs. JAMA 2014;311:1998-2006.

3. Mok TS, Wu YL, Thongprasert S, et al. Gefitinib or carboplatin-paclitaxel in pulmonary adenocarcinoma. $\mathrm{N}$ Engl J Med 2009;361:947-57.

4. Peters S, Camidge DR, Shaw AT, et al. Alectinib versus Crizotinib in Untreated ALK-Positive Non-Small-Cell Lung Cancer. N Engl J Med 2017;377:829-38.

5. Shaw AT, Yeap BY, Solomon BJ, et al. Effect of crizotinib on overall survival in patients with advanced non-smallcell lung cancer harbouring ALK gene rearrangement: a retrospective analysis. Lancet Oncol 2011;12:1004-12.

6. Linardou H, Dahabreh IJ, Kanaloupiti D, et al. Assessment of somatic k-RAS mutations as a mechanism associated with resistance to EGFR-targeted agents: a systematic review and meta-analysis of studies in advanced non-smallcell lung cancer and metastatic colorectal cancer. Lancet Oncol 2008;9:962-72.

7. Aberle DR, Adams AM, Berg CD, et al. Reduced lungcancer mortality with low-dose computed tomographic screening. N Engl J Med 2011;365:395-409.

8. Chang B, Hwang JH, Choi YH, et al. Natural history of pure ground-glass opacity lung nodules detected by lowdose CT scan. Chest 2013;143:172-8.

9. Hansell DM, Bankier AA, MacMahon H, et al. Fleischner Society: glossary of terms for thoracic imaging. Radiology 2008;246:697-722.

10. Kim EA, Johkoh T, Lee KS, et al. Quantification of ground-glass opacity on high-resolution CT of small peripheral adenocarcinoma of the lung: pathologic and prognostic implications. AJR Am J Roentgenol 2001;177:1417-22.

11. MacMahon H, Naidich DP, Goo JM, et al. Guidelines for Management of Incidental Pulmonary Nodules Detected on CT Images: From the Fleischner Society 2017. Radiology 2017;284:228-43.

12. Choi H, Yang B, Nam H, et al. Population-based prevalence of bronchiectasis and associated comorbidities in South Korea. Eur Respir J 2019;54:1900194.

13. Kim TJ, Lee CT, Jheon SH, et al. Radiologic Characteristics of Surgically Resected Non-Small Cell 
Lung Cancer With ALK Rearrangement or EGFR

Mutations. Ann Thorac Surg 2016;101:473-80.

14. Liu Y, Kim J, Qu F, et al. CT Features Associated with Epidermal Growth Factor Receptor Mutation Status in Patients with Lung Adenocarcinoma. Radiology 2016;280:271-80.

15. Snoeckx A, Reyntiens P, Desbuquoit D, et al. Evaluation of the solitary pulmonary nodule: size matters, but do not ignore the power of morphology. Insights Imaging 2018;9:73-86.

16. Detterbeck FC, Boffa DJ, Kim AW, et al. The Eighth Edition Lung Cancer Stage Classification. Chest 2017;151:193-203.

17. Travis WD, Brambilla E, Noguchi M, et al. International association for the study of lung cancer/american thoracic society/european respiratory society international multidisciplinary classification of lung adenocarcinoma. J Thorac Oncol 2011;6:244-85.

18. Lee T, Lee B, Choi YL, et al. Non-small Cell Lung Cancer with Concomitant EGFR, KRAS, and ALK Mutation: Clinicopathologic Features of 12 Cases. J Pathol Transl Med 2016;50:197-203.

19. Paik JH, Choe G, Kim H, et al. Screening of anaplastic lymphoma kinase rearrangement by immunohistochemistry in non-small cell lung cancer: correlation with fluorescence in situ hybridization. J Thorac Oncol 2011;6:466-72.

20. Ilie MI, Bence C, Hofman V, et al. Discrepancies between FISH and immunohistochemistry for assessment of the ALK status are associated with ALK 'borderline'-positive rearrangements or a high copy number: a potential major issue for anti-ALK therapeutic strategies. Ann Oncol 2015;26:238-44.

21. Cabillic F, Hofman P, Ilie M, et al. ALK IHC and FISH discordant results in patients with NSCLC and treatment response: for discussion of the question-to treat or not to treat? ESMO Open 2018;3:e000419.

22. Collett D. Modelling survival data in medical research: CRC Press; 2015.

23. Sabri A, Batool M, Xu Z, et al. Predicting EGFR mutation status in lung cancer:Proposal for a scoring model using imaging and demographic characteristics. Eur Radiol 2016;26:4141-7.

24. Yano M, Sasaki H, Kobayashi Y, et al. Epidermal growth factor receptor gene mutation and computed tomographic findings in peripheral pulmonary adenocarcinoma. J Thorac Oncol 2006;1:413-6.

25. Glynn C, Zakowski MF, Ginsberg MS. Are there imaging characteristics associated with epidermal growth factor receptor and KRAS mutations in patients with adenocarcinoma of the lung with bronchioloalveolar features? J Thorac Oncol 2010;5:344-8.

26. Chung JH, Choe G, Jheon S, et al. Epidermal growth factor receptor mutation and pathologic-radiologic correlation between multiple lung nodules with groundglass opacity differentiates multicentric origin from intrapulmonary spread. J Thorac Oncol 2009;4:1490-5.

27. Wang T, Zhang T, Han X, et al. Impact of the International Association for the Study of Lung Cancer/ American Thoracic Society/European Respiratory Society classification of stage IA adenocarcinoma of the lung: Correlation between computed tomography images and EGFR and KRAS gene mutations. Exp Ther Med 2015;9:2095-103.

28. Wang H, Schabath MB, Liu Y, et al. Clinical and CT characteristics of surgically resected lung adenocarcinomas harboring ALK rearrangements or EGFR mutations. Eur J Radiol 2016;85:1934-40.

29. Colen R, Foster I, Gatenby R, et al. NCI Workshop Report: Clinical and Computational Requirements for Correlating Imaging Phenotypes with Genomics Signatures. Transl Oncol 2014;7:556-69.

30. Fukui T, Yatabe Y, Kobayashi Y, et al. Clinicoradiologic characteristics of patients with lung adenocarcinoma harboring EML4-ALK fusion oncogene. Lung Cancer 2012;77:319-25.

31. Nakada T, Okumura S, Kuroda H, et al. Imaging Characteristics in ALK Fusion-Positive Lung

Adenocarcinomas by Using HRCT. Ann Thorac Cardiovasc Surg 2015;21:102-8.

32. NCCN Clinical Practice Guidelines in Oncology, NonSmall Cell Lung Cancer Version 6.2020. Available online: https://www.nccn.org/professionals/physician_gls/pdf/ nscl.pdf

33. Rizzo S, Petrella F, Buscarino V, et al. CT Radiogenomic Characterization of EGFR, K-RAS, and ALK Mutations in Non-Small Cell Lung Cancer. Eur Radiol 2016;26:32-42.

34. Han HS, Lim SN, An JY, et al. Detection of EGFR mutation status in lung adenocarcinoma specimens with different proportions of tumor cells using two methods of differential sensitivity. J Thorac Oncol 2012;7:355-64.

35. Lee H, Joung JG, Shin HT, et al. Genomic alterations of ground-glass nodular lung adenocarcinoma. Sci Rep 2018;8:7691.

36. Isaka M, Kojima H, Takahashi S, et al. Risk factors for local 
recurrence after lobectomy and lymph node dissection in patients with non-small cell lung cancer: Implications for adjuvant therapy. Lung Cancer 2018;115:28-33.

37. Lopez Guerra JL, Gomez DR, Lin SH, et al. Risk factors

Cite this article as: Choi $\mathrm{Y}$, Kim KH, Jeong BH, Lee KJ, Kim H, Kwon OJ, Kim J, Choi YL, Lee HY, Um SW. Clinicoradiopathological features and prognosis according to genomic alterations in patients with resected lung adenocarcinoma. J Thorac Dis 2020;12(10):5357-5368. doi: $10.21037 /$ jtd-20-1716 for local and regional recurrence in patients with resected N0-N1 non-small-cell lung cancer, with implications for patient selection for adjuvant radiation therapy. Ann Oncol 2013;24:67-74. 